\title{
Usability Evaluation of SMS-Based System: Basis for Systems Development
}

\author{
https://doi.org/10.3991/ijim.v13i09.10918 \\ Jake Guabes Maggay \\ Cagayan State University, Lasam, Philippines \\ jakegmaggay@gmail.com
}

\begin{abstract}
The objective of the study is to evaluate the usability of SMSBased Auto-Reply Inquiry System for Grades and Accounts. This descriptive study used a three-part questionnaire which was administered by the researcher. The usability questionnaire adapted from the USE Questionnaire used a 5-point scale composed of 30 statements. Convenience sampling was used choosing the respondents from the total population of 149 students of the CICS during the second semester of school year 2018-2019. A total of 45 questionnaires were retrieved and were used in the usability analysis. The usability evaluation results yielded a grand mean of 4.54 (Strongly Agree) showing that the SMS-based inquiry system is easy to learn, easy to use, useful, and satisfying. The results of this study show that pre-coded keywords are confusing for beginners but can be learned and familiarized by the users as they continue use the system. Further investigation should be conducted to prove that ease of use, ease of learning, and usefulness have direct impacts to user satisfaction.
\end{abstract}

Keywords-Auto-reply system; SMS-based system; system performance; usability; USE questionnaire

\section{$1 \quad$ Introduction}

\subsection{Background}

Short Message Service (SMS) is one of the technologies being used by organizations especially in processing, retrieving and sharing of relevant information. It is one of the features and basic service of mobile phones which allows the exchange of text messages between subscribers. SMS messages used Short Message Service Center (SMSC) which is being maintained by the cellular providers which enables mobile phones to send text messages up to 160 characters. It is evident that SMS messaging is becoming a widely used medium of communication for numerous mobile phone users [1]. Nowadays, there have been several usages of mobile phones to provide health, banking and educational services which have various forms such as but not limited to mobile learning, information fetching and grade checking [2].

Unlike other universities, the Cagayan State University - Lasam Campus (CSULasam) is not utilizing a web-based application to provide access on grades and ac- 
counts of the students because of its geographical constraints which hinder the penetration of internet connection in most area of the municipality. To have an alternative mechanism in providing prompt service especially in accessing the grades and accounts of students, a SMS-Based Auto-Reply Inquiry System for Grades and Accounts called "InquiText" is being used at CSU-Lasam. The system is programmed to automatically reply inquiries from the users especially the students regarding their grades and accounts within the country. The system architecture is shown in Fig. 1 while the main system's interface (for admin user) is shown in Fig 2.

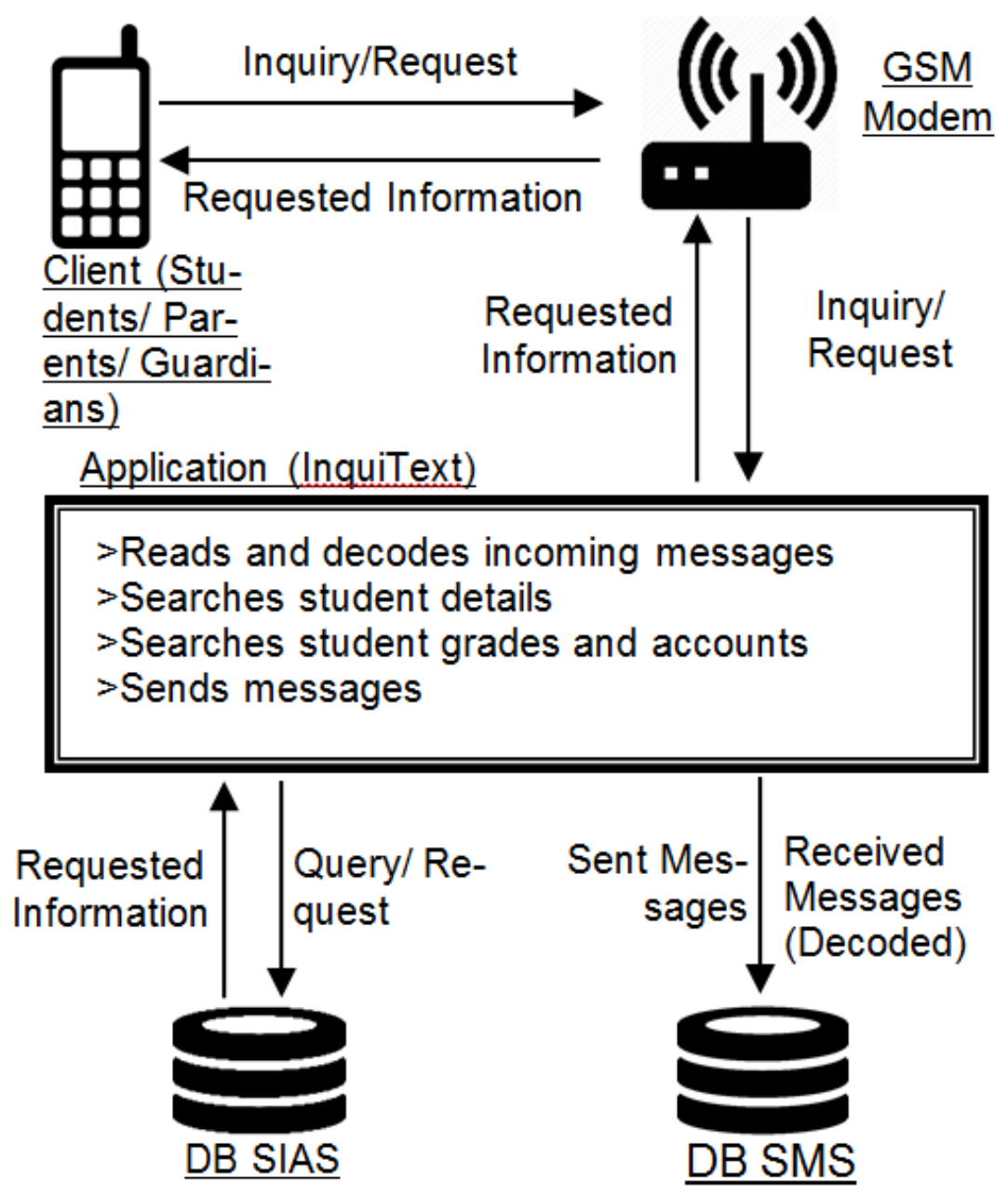

Fig. 1. System Architecture

Students and even guardians can inquire grades and accounts by sending keywords - in the form of SMS - to the cell phone number installed in the GSM (Global System for Mobile Communication Standards) modem which is being maintained by the 
school. These keywords are composed of different parts such as student number, school year and semester (Fig. 3 and 4). Upon sending the keyword, the system will automatically reply the user based on what is being inquired or requested. Aside from inquiry of grades and accounts as the system's main features, students can also register their cell phone numbers for them to receive regular announcements and updates from the university (Fig. 5). Notification will also be sent to the users if the syntax of the pre-coded keywords was not followed (Fig. 6). Moreover, registered numbers and all responses sent by the system are stored in a database.

Meanwhile, usability is defined based on ISO 9421-11 as "The extent to which a product can be used by specified users to achieve specified goals with effectiveness, efficiency and satisfaction in a specified context of use" [3]. In various applications, usability consists of usefulness and ease of use which are correlated. These factors are the drivers of user satisfaction and frequency of use [4]. In some studies, usability testing is use to provide guidelines in improving ease of use of products [5]. It was also emphasized that the overall goal of usability test is "to identify and rectify usability deficiencies existing in computer-based and electronic equipment" (Rubin, 1994 cited in [5]). Moreover, usability can be measured through usefulness, satisfaction and ease of use [6].

There are various studies on the usability of web and mobile applications with different usability models [7] but there is limited number of studies on the usability of SMS-based systems. This gap will be addressed by the study. The result of the study will be of great importance in the improvement and sustainability of the SMS-based inquiry system and in optimizing its use while continuously providing prompt and satisfactory service to the users. Moreover, the result will be used as basis for system developers in developing SMS-Based systems and other similar systems

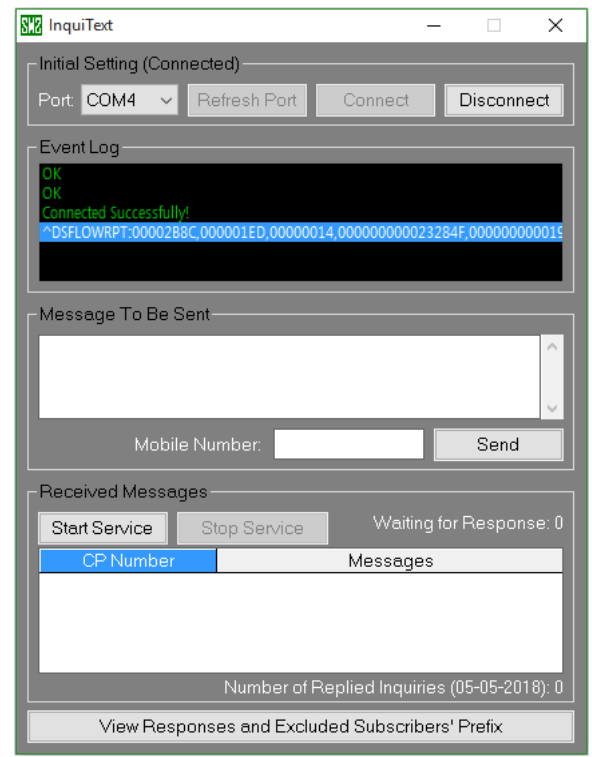

Fig. 2. System's Main Interface 


\section{5-27112 2017-20181}

Hi ERLE ANNE, here is the list of your grades:

IT 301 - 98 (Passed)

IT 302 - 98 (Passed)

IT 303 - 95 (Passed)

RES 11 - 96 (Passed)

SoC SCl 12 - 95 (Passed)

SOC SCl 13 - 95 (Passed)

PSYCHO 11 - 93 (Passed)

General Average - 95.71

Thank you for using InquiText.

Have a great day!

Fig. 3. Sample keyword and response of the system for inquiry of grades

\section{BAL 15-27112 2017-2018 1}

Name: ERLE ANNE G. GARCIA

Semester: First

SY: 2017-2018

Total Fees: $1,675.00$

Total Payment: $1,675.00$

Total Balance: 00.00

Thank you for using InquiText.

Have a great day!

Fig. 4. Sample keyword and response of the system for inquiry of accounts

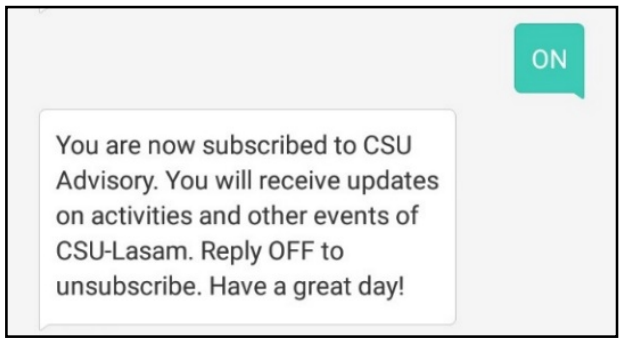

Fig. 5. Sample keyword and response of the system for subscription to CSU advisory 


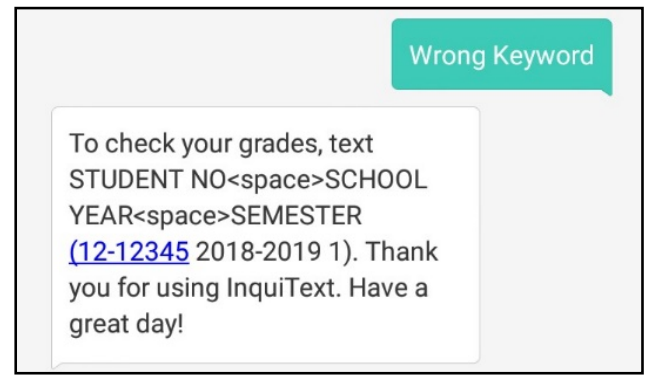

Fig. 6. Sample response of the system for incorrect keyword

\section{Objectives of the Study}

The objective of the study is to evaluate the usability of the SMS-Based AutoReply Inquiry System for Grades and Accounts of CSU-Lasam. It aims to answer the following questions. What is the usability level of the SMS-based inquiry system as to usefulness, ease of use, ease of learning, and user satisfaction? What are the advantages of the system to the students? What are the problems encountered by the students in using the system? What are the implications for system developers?

\section{$3 \quad$ Related Literature}

Usability of mobile applications focuses on visuals such as the screens, menus and buttons [8]. However, the SMS-based system to be evaluated in this study does not include screens, menus and buttons to be used by the users. The system interacts with the users through the use of text message containing pre-coded keywords (Fig. 3 to Fig. 6).

Usability is defined as "the quality of a user's experience when interacting with products or systems, such as websites, software, devices, or applications. It is about effectiveness, efficiency and the overall satisfaction of the user" [9]. It is also a step to ensure the quality of a product [10]. But some studies mentioned that metrics in measuring the effectiveness, efficiency and satisfaction may undue emphasis on speed and accuracy [11]. This study is very much concerned with the accuracy especially that grades and accounts being accessed by the students are very important.

In measuring evaluating usability, Nielsen's model of usability has five attributes such as efficiency, satisfaction, learnability, memorability, and errors. Efficiency deals with the resources such as time in relation to the accuracy and completeness of the goals while satisfaction refers to the positive feelings and attitudes towards the product. Learnability means that the system is easy to learn, while memorability refers to the attribute of the system which is easy to remember after a period of not being used. And lastly, errors refer to the number of errors that the user can make while using the system. In addition, the system should let the users recover after making some errors [7]. However, errors cannot be applied in the evaluation of the system 
because it does not have any screens that can be navigated, controlled or browsed by the users like websites and mobile applications.

To measure the Utilitarian and Experiential domain of usability, a study proposes the USE (Usefulness, Satisfaction, and Ease of use) questionnaire in measuring usability. In addition, this measuring tool measures the usability based on system's performance without ignoring the experience of the user [6]. Perceived usefulness is defined as "the degree to which a person believes that using a particular system would enhance his or her job performance" while perceived ease-of-use is defined as "the degree to which a person believes that using a particular system would be free from effort" (Davis, 1989 cited in [12]). A study shows that perceptions of usefulness particularly ease of use play an important role in the adoption and use of systems [12].

End-user satisfaction defined as "the overall cognitive and affective evaluation of the pleasurable level of consumption-related fulfilment experienced with the system". It is also believed that satisfaction of the users is one of the most essential determinants in the success of a system [13]. Conversely, Bevan, et al. [3] defined satisfaction as "positive attitudes, emotions or comfort resulting from use of a system, product or service". End-user satisfaction has also been studied in relation to other Information System aspects such as user acceptance, user resistance and system usability [14]. It is therefore necessary to include end-user satisfaction as one of the major determinants of system's usability, acceptance and its success.

Ease of learning or "learnability" emphasized that "the system should be easy to learn by the class of users for whom it is intended". For specific classification of learning, initial learning described as "allowing users to reach a reasonable level of usage proficiency within a short time" and extended learning is defined as "ease at which new users can begin effective interaction and achieve maximal performance" [15]. In addition, initial learning does not focus much on user performance. It focuses more on the level of proficiency with respect to time. A system's learnability is said to be in high level when a group of users can easily learn how to use it.

Meanwhile, mobile phones especially smartphones can be used for learning. The study of Papadakis, et al. [16] focuses on the use of Moodle to create flexibility in the learning practice of the students which is similar to one of the intentions of the SMSbased system being evaluated in this study. Likewise, their study establishes an interactive learning process between the teachers and the students. To determine the students' perception and use of the LMS, an evaluation was also conducted by Papadakis, et al. [17]. The result shows that students with longer engagement in using the Moodle have higher frequencies. Unfortunately, the general result of their study revealed that students do not prefer the use of mobile phones in using the Moodle. This study also evaluates the use of the SMS-Based System to determine its usefulness.

Most studies on usability evaluation focus on the software that facilitates the sending of SMS and do not focus on the interaction of the users to the system particularly on the responses being received by the users and keywords being sent by the users to the system. While some systems do not have the feature of receiving SMS from the users because they are only intended for sending of SMS. Examples of these studies are: a) the study of Lumauag [18] which aims to send notifications regarding school events and it was evaluated using ISO 9126-1; b) the studies of Mojares [19] which 
also aims to send SMS notification to parents regarding students' school attendance and it was evaluated using Hewlett-Packard methods; and other similar studies.

\section{$4 \quad$ Usability Framework}

The usability framework is anchored in the foregoing literature. Though, there are a lot of criteria for measuring usability, these set of criteria focus only on evaluating websites, computer software and systems, information systems and mobile phone applications. In addition, all of these include Graphical User Interface (GUI) being used by the users which does not exist in the SMS-based inquiry system particularly on the part of the users. The study adopted the three (3) dimensions of usability (usefulness, satisfaction, and ease of use) of the USE Questionnaire as basis in evaluating the usability level of the SMS-based inquiry system. The three dimensions are related in such that an improvement of ease of use improves usefulness and both dimensions are drivers for satisfaction. Moreover, ease of use can also be separated into two factors such as ease of learning and ease of use [20]. Conversely, usefulness, satisfaction, and ease of use were also recommended by Bringula and Basa [20] as part of usability evaluation. A derived usability framework from the foregoing literature is shown in Fig. 7.

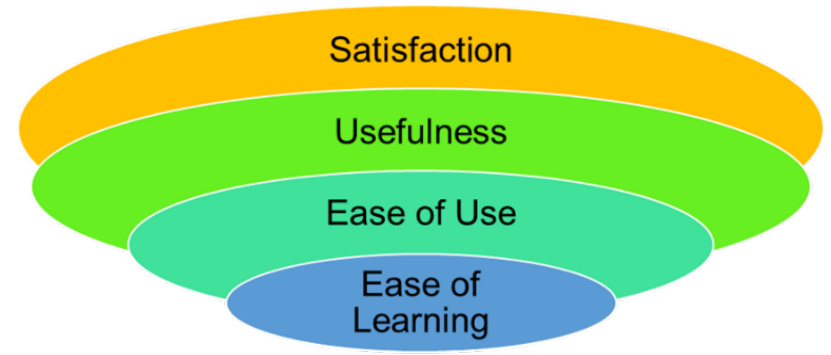

Fig. 7. Usability Framework

\section{$5 \quad$ Methodology}

This descriptive study used questionnaire which was administered by the researcher. A permission letter was sent to the college dean of the College of Information and Computing Sciences (CICS). Convenience sampling was used choosing the respondents from the total population of 149 students of the CICS during the second semester of school year 2018-2019. Though most of the respondents have already used the system, they were asked to inquire grades and accounts using their mobile phones before the questionnaires were distributed. A total of 45 questionnaires were retrieved and were used in the usability analysis.

The study used a three-part questionnaire. The first part is a self-made questionnaire for the basic profile of the respondents. The second part contains the usability questionnaire and composed of 30 statements using a 5-point scale adapted from the 
USE Questionnaire in the article of Yeap [4]. All statements measured ranging from "1 - Strongly Disagree" to "5- Strongly Agree" as shown Table 1.

Table 1. The 5-Point Scale and Its Verbal Interpretation and Mean Range

\begin{tabular}{|l|c|c|}
\hline \multicolumn{1}{|c|}{ Verbal Interpretation } & Weight & Mean Range \\
\hline Strongly Agree & 5 & $4.51-5.00$ \\
\hline Agree & 4 & $3.51-4.50$ \\
\hline Undecided & 3 & $2.51-3.50$ \\
\hline Disagree & 2 & $1.51-2.50$ \\
\hline Strongly Disagree & 1 & $1.00-1.50$ \\
\hline
\end{tabular}

The usability questionnaire is composed of four (4) sub-parts. The first sub-part is for Usefulness which has eight (8) statements. Ease of Use is the second sub-part that has eleven (11) statements. The third sub-part is the Ease of Learning which has four (4) statements. Satisfaction is the last sub-part of the usability questionnaire which has seven (7) statements with total of 30 usability statements (Table 2). The last part of the questionnaire is an open-ended questionnaire asking for the advantages and the problems encountered by the students in using the system.

Table 2. Composition of the Usability Questionnaire

\begin{tabular}{|l|c|}
\hline \multicolumn{1}{|c|}{ Dimensions of Usability } & Number of Statements \\
\hline Usefulness & 8 \\
\hline Ease of Use & 11 \\
\hline Ease of Learning & 4 \\
\hline Satisfaction & 7 \\
\hline
\end{tabular}

The profile of the respondents was described using frequency count and percentage while mean was used to determine the average scores of usability test of the system.

\section{$6 \quad$ Results and Discussion}

This section presents the profile of the responds, the usability level of the system, the advantages and problems encountered, and implication of the results to system developers.

\subsection{Respondents' profile}

Most of the respondents are female with frequency count of 28 or 62.22 percent of the total respondents while there are 17 male respondents or 37.78 percent of the total respondents. In terms of the type of mobile phones used during the testing, Table 3 shows that 12 respondents used basic phones and 12 respondents also used feature phones while 21 respondents used smartphones. This implies that inquiry of grades and accounts can be done through the use of any SMS-enabled mobile phone. In here, the system considered that not all students are using feature phone or smartphone (Fig. 8). 
Table 3. Type of Mobile Phone Used During Testing

\begin{tabular}{|l|c|c|}
\hline \multicolumn{1}{|c|}{ Type of Mobile Phone } & Count & Percentage \\
\hline Basic Phone (used mainly for making calls and texts) & 12 & 22.67 \\
\hline Feature Phone (texting, multimedia, a full web browser and GPS) & 12 & 22.67 \\
\hline Smartphone (performs many of the functions of a computer) & 21 & 46.66 \\
\hline
\end{tabular}

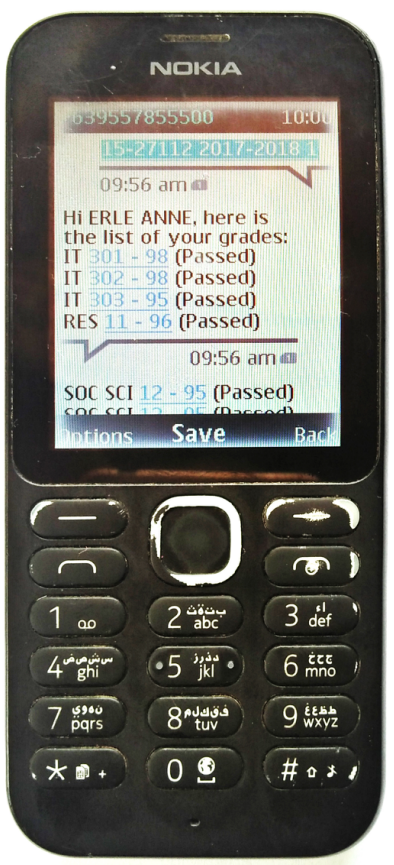

Fig. 8. Sample basic phone with sent keyword and received response

\subsection{Usability level}

Table 4. Usability Level of the SMS-Based Inquiry System

\begin{tabular}{|l|c|l|}
\hline \multicolumn{1}{|c|}{ Dimensions of Usability } & Mean & \multicolumn{1}{c|}{ Verbal Interpretation } \\
\hline Useful (Usefulness) & 4.58 & Strongly Agree \\
\hline Easy to Use (Ease of Use) & 4.51 & Strongly Agree \\
\hline Easy to Learn (Ease of Learning) & 4.47 & Agree \\
\hline Satisfying (Satisfaction) & 4.61 & Strongly Agree \\
\hline Grand Mean & 4.54 & Strongly Agree \\
\hline
\end{tabular}

As seen in Table 4, Ease of Learning got the lowest level among the four dimensions of usability which has a mean rating of 4.47 . However, the mean rating (mean = 4.47) still shows that the respondents can learn the system easily based on its verbal interpretation. The specific statement on the learnability of pre-coded keywords got the lowest mean rating in this dimension (mean $=4.38$ ). This is because the students need to memorize the syntax or format of the pre-coded keywords to be sent. For the 
inquiry of grades, students need to send their student number, followed by the school year and the specific semester as shown in Figure 3. Similarly, if the students will inquire on their accounts or balance, they need to send pre-coded keywords (BAL, student number, school year and specific semester) as shown in Figure 4. Moreover, students need to send ON to subscribe to CSU Advisory as shown in Figure 5. In the follow-up question, it shows that only those beginners have encountered difficulties during their first try and became proficient after a short period of using the system. This is similar to the statement mentioned by Grossman, et al. [21] about proficiency of users in using a system with respect to time.

Table 4 also shows that Satisfaction got the highest level among the four dimensions of usability with a mean rating of 4.61. Having the mean rating of 4.61 (Strongly Agree) implies that the respondents are satisfied with the SMS-based inquiry system. The specific statement in this dimension about the pleasantness in using the system got the highest mean rating (mean $=4.73$ ). Moreover, Ease of Use got a mean rating of 4.51 (Strongly Agree) while Usefulness got a mean rating of 4.58 (Strongly Agree) which means that the SMS-Based inquiry system is very easy to use and very useful.

Meanwhile, among the 30 statements from the different dimensions of usability, two statements got the highest mean rating of 4.96 (Strongly Agree). One of these is the statement on accuracy and reliability of response under Usefulness dimension. This is because data being accessed and sent to the students are directly from the school's database. In addition, the system has been implemented and tested for more than a year before the evaluation. Consequently, the system's bugs have been fixed during this period. Another statement having the highest mean rating is on flexibility (any phone can be used) under Ease of Use dimension. The system only sends purely texts which does not require high specifications of mobile phones. This means that any SMS-enabled mobile phone can be used to send and receive messages (SMS). Accordingly

Having a grand mean of 4.54 (Strongly Agree) shows that the SMS-based inquiry system is easy to learn, easy to use, useful, and satisfying. Moreover, the overall result attested that ease of use and usefulness are correlated in that an improvement of ease of use improves usefulness and both dimensions are drivers for satisfaction as mentioned by Lund [22] and Yeap [4]. It is also believed that learning the use of precoded keywords (Ease of Learning) will only be done for beginners but as they keep on using the system, it will become easy for them to use it (Ease of Use). Besides, the system provides instructions if the pre-coded keywords from the students do not conform the correct syntax as shown in Figure 6. If the students perceived its advantages, they will see the system as useful (Usefulness) which will result to their overall Satisfaction.

\subsection{Advantages and problems encountered}

This portion was obtained from the last part of the questionnaire which is an openended questionnaire asking for the advantages and the problems encountered by the students in using the system. It was mentioned by the respondents that the system 
makes the inquiry of grades and accounts very easy because according to them, they usually inquire when they are home or during vacation period. Because of this, they have mentioned that there is no need for them to travel and go to school just to check their grades. Furthermore, they have mentioned that the system is very useful and saves their time and effort in checking their grades. These advantages are supported by the mean rating of the specific statements from the four dimensions of usability.

However, there are some problems encountered by the respondents. Most of them mentioned that sometimes, they receive late or they do not receive any reply from the system. Some reasons behind this problem are as follows: 1) grades are submitted and updated in the registrar's office every end of the semester; therefore, the system will only be activated by the registrar during this period; 2) only few or no students inquire grades and accounts in the mid of the semesters. Because of this pattern, the registrar intends not to activate the system; 3 ) the system is available only during office hours (Monday to Friday, 8:00AM to 5:00PM) except holidays; 4) because the SIM Card (Subscriber Identification Module) needs to be registered for a particular subscription of unlimited texts, the system can no longer give response if the subscription has been expired until the SIM Card will be registered again; and 5) the system is intended only for specific network subscribers; hence, other network subscribers will be filtered and will no longer receive any reply.

\subsection{Implications for SMS-based system developers}

Based on the specific statements having the lowest mean rating, it implies that developers should consider the pre-coded keywords to be used by the intended users. Keywords should be short and easy to remember. Moreover, instructions should also be provided to the users especially for beginners. Users should also be involved during the development phase to gather their inputs. Nevertheless, it is very important for system developers to fix bugs before implementing any systems because this will affect the satisfaction of the users.

\section{Conclusion}

The objective of the study is to evaluate the usability of the SMS-Based AutoReply Inquiry System for Grades and Accounts of CSU-Lasam. The results of this study show that pre-coded keywords except for the keyword for subscription to CSU Advisory (Figure 1-c) are confusing for beginners but can be learned and familiarized by the users as they continue use the system. The results also show that the system provides advantages to the users. In addition, the system is flexible because any SMSenabled mobile phone can be used. Moreover, the system can send very accurate and reliable grades and accounts. The overall results revealed that the system has a very high level of usability which means that it is easy to learn, very easy to use, very useful and very satisfying. It also adds body of knowledge in filling the gap in the literature particularly on evaluation of SMS-based systems wherein screens, menus and buttons to be used by the users are not included. 


\section{Recommendations}

Based on the findings and some problems identified in this study, the researcher has the following recommendations:

- The system should always be active even during the mid of semesters to have a continuous service

- Parents or guardians should be informed about the existence and use of the SMSbased inquiry system for them to monitor the grades and accounts of their children and to maximize its use

- To avoid inconvenience of the users especially in not receiving any response from the system (when the subscription expires), a post-paid plan from a mobile network should be availed or used

- The result of this study yielded a high level of usability, thus, the school administration should continue to support the use of the SMS-based inquiry system and it must be maintained and sustained

- Though the study revealed that satisfaction got the highest rating, further investigation should be conducted to prove that ease of use, ease of learning, and usefulness have direct impacts to user satisfaction.

\section{$9 \quad$ References}

[1] J. Brown, B. Shipman and R. Vetter, "SMS: The Short Message Service," Computer, vol. 40, no. 12, p. 106, 2007. https://doi.org/10.1109/mc.2007.440

[2] T. Bhuiyan, I. Mahmud and B. Alam, "Usability Analysis of SMS Alert System for Immunization in the Context of Bangladesh," International Journal of Research in Engineering and Technology, vol. 2, no. 11, p. 300, 2013.

[3] N. Bevan, T. Geis, J. Earthy, G. Thomas and J. Carter, "New ISO Standards for Usability, Usability Reports and Usability Measures," 2016. https://doi.org/10.1007/978-3-319$\underline{39510-4 \quad 25}$

[4] M. Yeap, "Measuring Usability with the USE Questionnaire," 2009.

[5] J. Lewis, "Usability Testing," in Handbook of Human Factors and Ergonomics, New Jersey, John Wiley and Sons, Inc., 2012, p. 1267.

[6] T. K. Chung and N. Sahari, "Utilitarian or Experiential? An Analysis of Usability Questionnaire," International Journal of Computer Theory and Engineering, vol. 7, no. 2, pp. 168-171, April 2015.

[7] R. Harrison, D. Flood and D. Duce, "Usability of mobile applications: literature review and rationale for a new usability model," Journal of Interaction Science, vol. 1, no. 1, 2013. https://doi.org/10.1186/2194-0827-1-1

[8] M. Masood and M. Thigambaram, "The Usability of Mobile Applications for Preschoolers," Procedia - Social and Behavioral Sciences, pp. 1818-1826, 2015. https://doi.org/10.1016/j.sbspro.2015.07.241

[9] "Usability.gov," n.d.. https://www.usability.gov/what-and-why/usability-evaluation.html. [Accessed 23 December 2018].

[10] A. Lund, "Post-Modern Usability," Journal of Usability Studies, vol. 2, no. 1, pp. 1-6, 2006. 
[11] A. Dillon, "Beyond Usability: Process, Outcome and Affect in Human Computer Interactions," n.d..

[12] D. Shen, J. Laffrey, T. Lin and H. Huang, "Social Influence for Peceived Usefulness and Ease-of-Use of Course Delivery Systems," Journal of Interactive Online Learning, vol. 5, no. 3, pp. 270-282, 2006.

[13] K. Matusiak, "Perceptions of Usability and Usefulness of Digital Libraries," International Journal of Humanities and Arts Computing, vol. 6, no. 1-2, pp. 133-147, 2012. https://doi.org/10.3366/ijhac.2012.0044

[14] V. Aggelidis and P. Chatzoglou, "Hospital information systems: Measuring end user computing satisfaction (EUCS)," Journal of Biomedical Informatics, pp. 566-579, 2012. https://doi.org/10.1016/i.jbi.2012.02.009

[15] F. Karimi, D. Poo and Y. M. Tan, "Clinical information systems end user satisfaction: The expectations and needs congruencies effects," Journal of Biomedical Informatics, pp. 342354, 2015. https://doi.org/10.1016/i.jbi.2014.12.008

[16] S. Papadakis, M. Kalogiannakis, E. Sifaki and N. Vidakis, "Access Moodle Using Smart Mobile Phones. A case study in a Greek University," in Lecture Notes of the Institute for Computer Sciences, Social Informatics and Telecommunications Engineering, Switzerland, Cham, 2017. https://doi.org/10.1007/978-3-319-76908-0 36

[17] S. Papadakis, M. Kalogiannakis, E. Sifaki and N. Vidakis, "Evaluating Moodle use via Smart Mobile Phones. A case study in a Greek University.," EAI (European Alliance for Innovation) Endorsed Transactions on, vol. 5, no. 16, pp. 1-12, 2019. https://doi.org/10. 4108/eai.10-4-2018.156382

[18] R. Lumauag, "SENT SMS: School Event Notification Through SMS," Asia Pacific Journal of Multidisciplinary Research, vol. 4, no. 4, pp. 61-68, 2016.

[19] P. W. Mojares, "iNotified: An SMS and FRID-Based Notification System of Lipa City Colleges, Lipa City, Batangas Philippines," in International Conference on Computing, ELearning and Emerging Technology \& Inernational Conference on Advances in Computer, Electrical and Electronic Engineering, Sidney, Australia, n.d.. https://doi.org/10.20319 /pijss.2018.33.12621274

[20] R. P. Bringula and R. S. Basa, "Factors Affecting Faculty Web Portal Usability," Educational Technology \& Society, vol. 14, no. 4, pp. 253-265, 2011.

[21] T. Grossman, G. Fitzmaurice and R. Attar, "A Survey of Software Learnability: Metrics, Methodologies and Guidelines," Toronto, 2009.

[22] A. Lund, "Measuring Usability with the USE Questionnaire," Usability Interface, vol. 8, no. 2, pp. 3-6, 2001.

\section{Author}

Jake G. Maggay is a member of the National Research Council of the Philippines. $\mathrm{He}$ is an Instructor at the Cagayan State University - Lasam Campus under the College of Information and Computing Sciences. His research interests are information systems development, mobile technology and IT education.

Article submitted 2019-05-25. Resubmitted 2019-06-25. Final acceptance 2019-06-26. Final version published as submitted by the authors. 